\title{
THE GALACTIC CHEMICAL EVOLUTION OF LITHIUM
}

\author{
F. MATTEUCCI \\ Dipartimento di Astronomia, Universitá di Trieste \\ Via G. Tiepolo 11, Trieste, Italy
}

Under the assumption that the abundance of ${ }^{7} \mathrm{Li}$ in Population II stars represents the primordial Li abundance (with perhaps a small contribution from GCR spallation) and that GCR spallation/fusion processes cannot contribute to more than $\simeq 10-20 \%$ of the $\mathrm{Li}$ abundance observed in Pop. I stars and in the solar system, one must conclude that most of $\mathrm{Li}$ in Pop. I stars has a stellar origin.

Possible stellar Li producers are discussed: low mass AGB stars (2 $5 M_{\odot}$ ) (C-stars), high mass AGB stars $\left(5-8 M_{\odot}\right)$, supernovae of type II $\left(M>10 M_{\odot}\right)$ and novae. The various problems connected with all of these sources are indicated: in particular, we discuss the Li production in AGB stars when evolutionary effects due to the metallicity are taken into account, and the fact that novae do not seem to be good candidate for Li production, as suggested by a recent nucleosynthesis study. We then calculate the yields from these stellar sources and predict the behavior of $\log \mathrm{N}(\mathrm{Li}) \mathrm{vs}$. $[\mathrm{Fe} / \mathrm{H}]$ by means of a galactic chemical evolution model.

We conclude that, although a unique model cannot be found, due to the uncertainties still existing in the stellar nucleosynthesis, the most likely scenario is that $\mathrm{Li}$ is partly produced in type II supernovae ( $\nu$-induced nucleosynthesis) and partly in massive AGB stars. 